\title{
Updated Photonuclear Data Library and Database for Photon Strength Functions
}

\author{
Paraskevi Dimitriou $^{1, a}$, Richard B. Firestone ${ }^{2}$, Sunniva Siem ${ }^{3}$, František Bečvár̆ ${ }^{4}$, Milan Krtička ${ }^{4}$, Vladimir V. Varlamov ${ }^{5}$, and \\ Mathis Wiedeking ${ }^{6}$ \\ ${ }^{1}$ Nuclear Data Section, International Atomic Energy Agency, A-1400 Vienna, Austria \\ ${ }^{2}$ Lawrence Berkeley National Laboratory, University of California, CA 94720, USA \\ ${ }^{3}$ Department of Physics, University of Oslo, P.O. Box 1048 Blindern, N-0316 Oslo \\ ${ }^{4}$ Charles University in Prague, V Holesovickach 2, 18000 Prague, Czech Republic \\ ${ }^{5}$ CDFE, Skobeltsyn Institute of Nuclear Physics, Lomonosov Moscow State University, 119991 Moscow, Russia \\ ${ }^{6}$ iThemba LABS, P.O. Box 722, Somerset West 7129, South Africa
}

\begin{abstract}
Photonuclear cross sections and gamma-ray data used to extract Photon Strength Functions are important for a large range of applications including basic sciences. The recommendations of an IAEA Consultant's Meeting to update the IAEA Photonuclear Data Library and create a Reference Database for Photon Strength Functions are presented.
\end{abstract}

\section{Motivation}

Gamma-ray data from nuclear reactions are important for a large range of applications, ranging from energy, safety and medical applications to nuclear physics and astrophysics.

A wealth of gamma-ray data related to photonuclear reactions and Photon Strength Functions (PSF) accumulated in recent years need to be made available to researchers worldwide. These data are important sources of information for experimental data files such as EXFOR and evaluated data files such as RIPL, ENDF, EGAF, ENSDF etc supported by the International Atomic Energy Agency. They are also intricately related to the development and improvement of theoretical models describing the electromagnetic response of the nucleus.

The current situation with regards to photonuclear and reaction gamma-ray data was reviewed at a Consultant's Meeting organized by the Nuclear Data Section IAEA in November 2013 [1].

In this paper we report on the main issues that were addressed at the meeting and the recommendations that were made.

\section{Overview of existing data}

\subsection{Photonuclear data}

A database of photonuclear data was generated under an IAEA CRP in 2000 [2] and includes $\gamma$ absorption data, total and partial photo-neutron reaction cross sections, and neutron emission energy spectra for 164 isotopes (from ${ }^{2} \mathrm{H}$ to ${ }^{241} \mathrm{Pu}$ ), primarily for structural, shielding, biological and fissionable materials.

Although this database has been extremely useful to a broad community, it is now evident that it needs to be revised especially since:

- Some of the data are unreliable and discrepant.

- For 37 isotopes $\left({ }^{3} \mathrm{H},{ }^{3} \mathrm{He},{ }^{6,7} \mathrm{Li},{ }_{10,11} \mathrm{~B},{ }_{14}^{14} \mathrm{C},{ }_{14}^{19} \mathrm{~F},{ }^{45} \mathrm{Sc}\right.$, ${ }^{75} \mathrm{As},{ }^{76,78,80,82} \mathrm{Se},{ }^{89} \mathrm{Y},{ }^{103} \mathrm{Rh},{ }_{153}^{115} \mathrm{In},{ }^{138} \mathrm{Ba},{ }_{160}^{139} \mathrm{La},{ }^{140,142} \mathrm{Ce}$, ${ }_{142,143,144,145,146,148,150} \mathrm{Nd}, \quad{ }^{153} \mathrm{Eu}, \quad{ }^{160} \mathrm{Gd}, \quad{ }^{175} \mathrm{Lu}$, $186,188,189,190,192$ Os, ${ }^{237} \mathrm{~Np}$ ) there exist data that have not been evaluated but can be used in basic research.

- There exist improved evaluation techniques.

- Many new data have become available in the recent years.

\subsection{Photon scattering cross-section data}

These data are important for the investigation of the PSF below the neutron separation energy in an energy region which is so far poorly explored. There has been an increase in new photon scattering cross-section data $\left(\gamma, \gamma^{\prime}\right)$ coming from recently commissioned facilities. Currently, there is no database that compiles these data for easy access by the user community.

\subsection{Charged-particle reaction data}

There has been a growth in the development of large detector arrays for the measurement of reaction $\gamma$ rays in coincidence with emitted charged particles. These

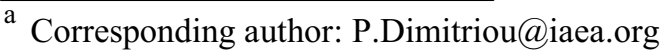


experiments provide a capability for investigating the PSF below the particle emission threshold. Although some data (obtained using the Oslo method) are made available through the Oslo group website [3], there is no dedicated database which provides all these data to the applications and basic research community.

\subsection{Neutron-capture data}

Recently there has been significant development of guided neutron beam facilities for the measurement of thermal and cold neutron-capture $\gamma$ rays. Primary $\gamma$ rays from these experiments have been compiled in the IAEA EGAF database [4] and can be useful in investigating the distribution of individual primary $\gamma$-ray strengths. These data are supplemented by average resonance-capture data and neutron time-of-flight data that provide additional measurements of photon strengths.

Additionally, coincidence measurements of $\gamma$ rays allow the investigation of the PSF in the so-far poorly explored quasi-continuum region. There are several experimental facilities where coincidence measurements of $\gamma$ rays are being performed. These data are complementary to the measurements mentioned above, but have not been compiled in a dedicated database so far.

\section{Experimental and evaluation methods}

The existing experimental and evaluation methods were reviewed and their advantages and drawbacks were assessed. In some cases the different experimental and evaluation methods give different results. These discrepancies need to be resolved before any set of data can be recommended for use in the various applications. Some examples of discrepancies are given in the following.

\subsection{Above particle threshold}

The situation above the particle threshold emission is illustrated in Figure 1. Here, one can see the discrepancies in the measured and evaluated cross sections for the photonuclear reaction ${ }^{197} \mathrm{Au}(\gamma, 2 \mathrm{n})$. The evaluation produced by the IAEA CRP on Photonuclear Data [2], using GUNF and GNASH codes to reproduce the Saclay ${ }^{197} \mathrm{Au}\left(\gamma, \mathrm{S}_{\mathrm{n}}\right)$ data [5], does not satisfy newly introduced reliability criteria which are based on multiplicity transitional functions $F_{i}-$ ratios of the definite partial reaction cross sections $\sigma(\gamma, i n)$ to the neutron yield reaction cross section $\sigma\left(\gamma, \mathrm{S}_{\mathrm{n}}\right)$.

For example, according to the definition $\mathrm{F}_{2}$ cannot exceed 0.50 in magnitude under any conditions: its value above this absolute limit would mean a physically incorrect determination of the $(\gamma, 2 n)$ and the corresponding $(\gamma, 1 \mathrm{n})$ reaction cross sections (see [1] and references therein).

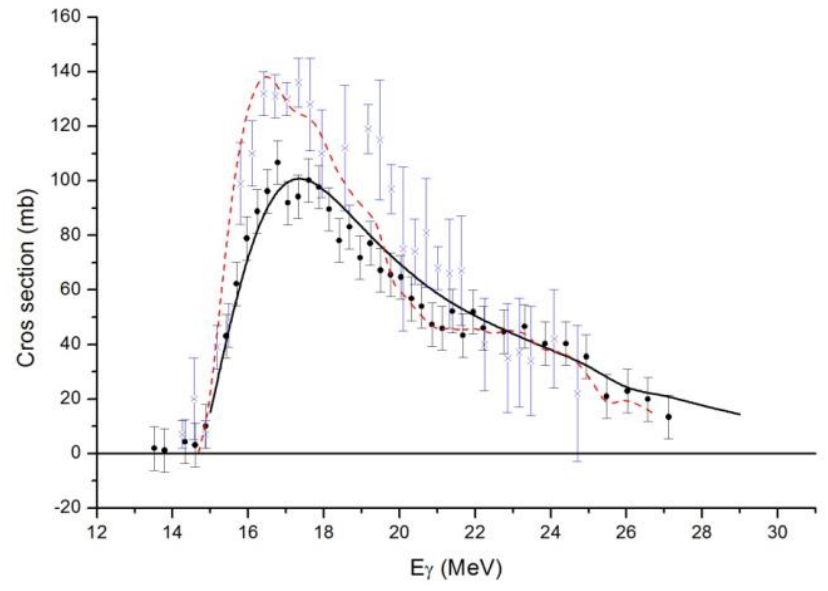

Figure 1. Comparison of experimental data from Saclay [5] (circles) and Livermore [6] (crosses) for ${ }^{197} \mathrm{Au}(\gamma, 2 \mathrm{n}){ }^{195} \mathrm{Au}$ reaction cross section with evaluated data (line - IAEA CRP, dotted line - new evaluation based on data reliability criteria $\mathrm{F}_{2}$ ).

\subsection{Below or near particle threshold}

The situation below or near the particle separation threshold is illustrated in Figure 2 for ${ }^{98} \mathrm{Mo}$ [7]: one can see the discrepant results from the different experimental methods used to determine PSFs.

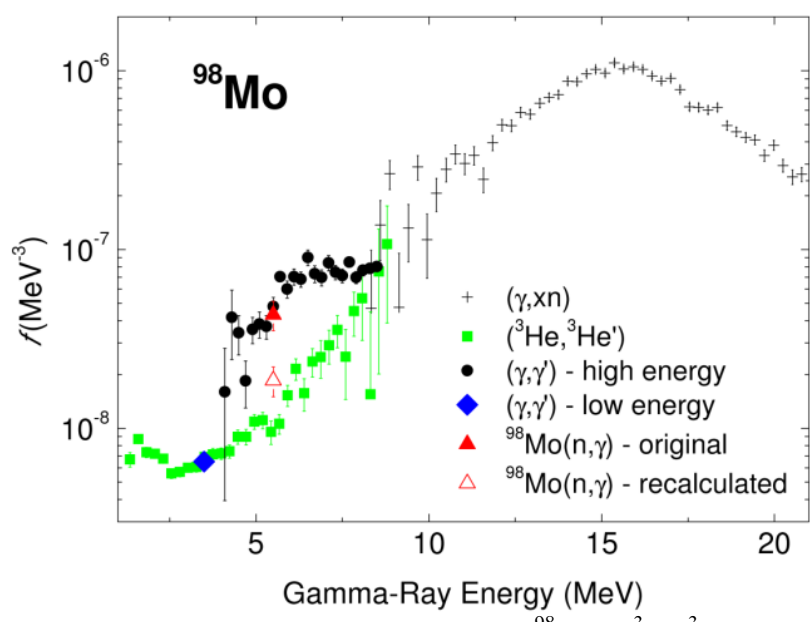

Figure 2. Available data on PSFs for ${ }^{98} \mathrm{Mo}:\left({ }^{3} \mathrm{He},{ }^{3} \mathrm{He}\right)$ [8], $\left(\gamma, \gamma^{\prime}\right)[9,10],(\gamma, \mathrm{xn})[11]$. In addition, data from intensities of primary transitions following radiative neutron capture in ${ }^{98} \mathrm{Mo}$ $[12,13]$ are shown. The figure was taken from Ref. [7].

The figure shows that PSFs derived from $\left({ }^{3} \mathrm{He},{ }^{3} \mathrm{He}\right.$ ') reaction data using the Oslo method (green squares) [8] display a very different shape compared to the strength function deduced from photon scattering cross sections measured using a Bremsstrahlung beam (black circles) [9]. It is interesting to note that the photon strength deduced from measurements of intensities of $\gamma$ transitions exciting levels near $3 \mathrm{MeV}$ [10] agrees with the $\left({ }^{3} \mathrm{He},{ }^{3} \mathrm{He}\right)$ data. In addition, there is also the problem with the photon strength derived from radiative neutron capture measurements on isolated resonances. The only difference in the two data from $(n, \gamma)$ is the assumed resonance spacing which was taken from two different 
sources, Ref. [12] for "original" and [13] for "recalculated" data. For details see Ref. [7].

Clearly there is a need to perform systematic intercomparisons of measurements for several isotopes in different mass regions to investigate the potential causes of these discrepancies. The development of a database dedicated to reaction $\gamma$-ray data would help such an effort.

\section{Database}

From the previous section it follows that there is a need for a new reference database for PSFs that would provide all the useful experimental and evaluation information, as well as recommendations to the user community.

The new database for PSFs should include a compilation of all existing data. It should be divided in sections corresponding to the different experiments (photon scattering, charged-particle $\gamma$-ray, neutroncapture primary/coincidence $\gamma$-ray data). Each dataset should include a short description of the experiment.

The detailed structure and format of this new database should be developed taking into consideration the ENSDF and RIPL evaluation efforts.

The methodology for the evaluation of the data should be clearly defined and evaluators selected on the basis of expertise in the various sections.

Current theoretical approaches should also be investigated and comparisons with data should be made available in the database.

\section{Conclusions}

After reviewing the current situation with regards to photonuclear data and reaction $\gamma$-ray data used for the determination of PSFs, the IAEA meeting made the following recommendations:

The IAEA Photonuclear Data Library should be updated to take into account new experimental data as well as new evaluation criteria.

A Reference Database should be generated for the compilation of all PSF data. The database should also provide recommended values to the user community.

The meeting concluded that a concerted effort under the coordination of the IAEA was the most effective way of implementing the above recommendations.

\section{References}

1. P. Dimitriou, R. Firestone, and S. Siem, Summary Report of Consultant's Meeting on Compilation and Evaluation of Gamma-ray Data, INDC(NDS)-0649, 4-6 November 2013, IAEA, Vienna.

http://www-nds.iaea.org/publications/inde/indc-nds0649.pdf

2. IAEA Photonuclear Data Library, http://www-nds.iaea.org/photonuclear/

3. Oslo group data, http://www.mn.uio.no/fysikk/english/research/about/
infrastructure/OCL/nuclear-physics research/compilation/

4. IAEA EGAF database, http://www-nds.iaea.org/pgaa/

5. A. Veyssiere et. al., Nucl. Phys. 159, 561 (1970)

6. S. C. Fultz et. al., Phys. Rev. 127, 1373 (1962)

7. M. Krticka and F. Becvar, EPJ Web of Conferences 2, 03002 (2010)

8. M. Guttormsen et al., Phys. Rev. C 71, 044307 (2005)

9. G. Rusev et al., Phys. Rev. C 77, 064321 (2008)

10. G. Rusev et al., Phys. Rev. C 73, 044308 (2006)

11. H. Beil et al., Nucl. Phys. A 227, 427 (1974)

12. J. Kopecky, M. Uhl, RIPL1, IAEA, Vienna, http://www-nds.iaea.org/RIPL-1/

13. T. Belgya et al., RIPL2, IAEA, Vienna, 2005. http://www-nds.iaea.org/RIPL-2/ 
